\title{
Pillañ peuma rruaín: el mapudungún en Chiloé a fines del siglo XIX según los collag
}

\section{Roberto Bahamonde Andrade ${ }^{*}$}

\begin{abstract}
Resumen
En este estudio se caracteriza la fonética-fonología, gramática y léxico del mapudungún empleado en los collag (cantos) recopilados en el mar interior de Chiloé por Juan Elías Necul alrededor de 1887, pocos años antes de la extinción local del idioma. El trabajo se centra en el sistema de consonantes, el inventario y función de los sufijos verbales y el vocabulario empleado en los cantos. A partir de los resultados se concluye que esta variedad no parecía compartir los rasgos diferenciales del chesüngun hablado actualmente en la región de Los Lagos, que los sufijos de las formas no finitas se habían lexicalizado o habian adoptado otras funciones y que la mayor parte del vocabulario no difiere del vocabulario corriente en otras variedades de la lengua mapuche.
\end{abstract}

Palabras clave: mapudungun, Chiloé, collag, veliche.

\section{Pillañ peuma rruaín: mapudungun in Chiloe Archipelago at late 19 th century according to collag}

\begin{abstract}
$^{1}$
In the present study, phonetics-phonology, grammar and vocabulary of Mapuche language used in collag (songs) are characterized. Consonant system, inventory and function of verbal suffixes and vocabulary were analyzed. From results arise three main conclusions: Firstly, this variety apparently did not share the specific features of chesüngun dialect, currently spoken in Los Lagos Region. Secondly suffixes of non-finite forms became lexicalized or changed irregularly their functions. Lastly, most of the vocabulary present in collag was the same as other varieties of Mapuche language.
\end{abstract}

Key words: Mapuche language, Chiloé, collag, veliche.

\footnotetext{
* Chileno, Licenciado en Agronomía Universidad Austral de Chile, investigador independiente. rbahamondea@gmail.com

1 Agradezco a Elizabeth Megas, por su revisión del texto en inglés.
} 


\section{Presentación}

\subsection{Mapudungun en Chiloé}

A mediados del siglo XVI, el mapudungún se hablaba entre el centro del actual territorio de Chile y el archipiélago de Chiloé (Zúñiga, 2006). De acuerdo a lo escrito por el gobernador Santa María en 1775 y por el geógrafo Moraleda en 1786, la población indígena y española era bilingüe, aunque para el final de ese siglo el castellano comenzaba a ganar terreno, debido en parte a disposiciones que buscaban la homogeneización lingüística de los habitantes de la provincia. En el siglo XIX, luego de la anexión a Chile, el proceso se aceleró (Cárdenas, Montiel y Hall, 1991).

Según Cavada (1914, p. 265), en su tiempo la variedad local del idioma, denominada "veliche", era una lengua poco usada, solo por ancianos, y la consideraba "próxima ya a desaparecer", mientras que Cañas (1908) afirma que podía considerarse muerta luego del fallecimiento de quienes eran capaces de usarla con fluidez.

En la década de 1930, llegó al sur de Chiloé el lonco Juan Fermín Lemuy Treumún de Osorno, quien ayudó a impulsar un movimiento de organización de las comunidades de la zona para la defensa de territorio y la cultura huilliches, que enfrentaban amenazas de empresas y latifundistas. Como parte de ese proyecto, en 1939 llegó Juan de Dios Cheuquián, para preparar a 12 niños y 12 niñas como maestros de paz y para que aprendieran la lengua mapuche en la variante de Osorno, que era la que él empleaba (Cárdenas, Montiel y Hall, 1991; Trivero, 1999).

Sin embargo, este trabajo de revitalización del idioma no revirtió la situación y a fines del siglo XX la población huilliche (de willi sur y che gente) del archipiélago tenía un conocimiento mínimo del mapudungún (Salas, 1992).

Existen pocos registros del mapudungún hablado en Chiloé, pues las obras creadas por misioneros durante el periodo colonial (1567-1826) están perdidas o destruidas. Un integrante de la expedición holandesa de Hendryk Brouwer a Chiloé y Valdivia de 1642-1643 recogió un vocabulario y frases en "lengua chilena", publicado en latín en 1647, pero no hay información acerca del origen de los datos (Schuller, 1907). El pequeño diccionario que elaboró en Chiloé el padre Gaspar López (1679-1724) fue una de las fuentes que usó Andrés Febrés al elaborar el Calepino ChilenoHispano de 1765 (Rondón, 2014). 


\subsection{Collag}

Los collag que se analizarán en este trabajo fueron recogidos a fines del siglo XIX, cuando el veliche ya no era la lengua cotidiana de la población. Su recopilador fue Juan Elías Carrera, cuyo apellido original era Necul (nekül, ágil), un hombre de la isla Cahuach que había desempeñado cargos públicos en el municipio de Quenac. Junto con los cantos, Necul dio información acerca de su ejecución, de su función como acompañamiento de medanes (fiestas para reunir materiales) y quemunes ("paseos" de vista entre compadres), e incluso los nombres de algunos de los romanceros que los cantaban en la isla de Apiao: Jacinto Millalonco, Valerio Millalonco, Noburga Peranchiguai e Irene Tureuna (Cañas, 1908).

Alejandro Cañas Pinochet publicó 31 cantos (collag) de Necul en "La poesía en los pueblos primitivos", texto que apareció por primera vez en la Revista Chilena de Historia Natural en 1908. En él, Cañas compara lo que considera diferentes formas de poesía de los pueblos indígenas de América, entre las que destaca especialmente a los collag (Cañas, 1908).

La ortografía del texto en mapudungún fue ideada por Necul, pero la traducción corresponde a Cañas, quien afirmó respetar la traducción de Necul": "aunque a veces la inspiracion me llevase a la espresion de un pensamiento que en mi sentir lo hacia mas poético i mas espresivo que el orijinal, refrenaba esa inspiracion" (Cañas, 1908, p. 134)

Contreras (2009) hace notar que "la traducción de los textos a ratos parece bastante libre, pero Cañas le reconoce rigor, porque fue un trabajo en que ambos (informante e investigador) aportaron su saber" (p. 319). Sin embargo, Rodolfo Lenz tuvo a la vista las traducciones originales de Necul, remitidas a él por Cañas alrededor de una década antes de su publicación, y afirma: "Por desgracia el señor Cañas Pinochet no ha acompañado su edicion de la traduccion mas o ménos literal que habia escrito el mismo indio, sino que ha trasformado esta en versos castellanos, que se apartan a menudo de un modo fantástico del sentido del orijinal" y "Las curiosas poesías apuntadas por Necul son dignas de una edicion mas fonética con traduccion ménos fantástica que la del señor Cañas Pinochet, pero la tarea no es fácil" (Lenz, 1912, p. 760). Estas apreciaciones de Lenz sobre los collag aparecieron en un artículo de 1912 sobre las tradiciones

2 Las citas textuales aparecen en su ortografía original, que puede tener diferencias con la ortografía actual. especialmente por el uso de la denominada "ortografía chilena", vigente en Chile entre 1844 y 1927. 
e ideas de los mapuches acerca de los terremotos, y el autor menciona el llamado Collag del terremoto, que según Cañas (1908) alude al terremoto de 1837, pero la traducción de Necul y el título original Entuhuampun ['Sacar un bongo'] dan a entender que en verdad es un canto acerca del arrastre de un bongo (canoa hecha de un tronco ahuecado) por una playa pedregosa (Lenz, 1912).

En 1911 Cañas publicó sus Estudios de la lengua veliche, que consisten en la fusión del artículo de 1908, de un diccionario publicado previamente y de otros materiales acerca del veliche. Dentro del Glosario de la lengua veliche aparece un collag adicional en las páginas 302 y 303, muy parecido a los primeros 6 versos del collag titulado "Segunda conversación", publicado en 1908 (Cañas, 1911).

El objetivo del estudio de estos collag es caracterizar el mapudungún que se hablaba o al menos cantaba en Chiloé a fines del siglo XIX. Para ello, se analizarán rasgos relevantes de la fonética, gramática y vocabulario que pueden colegirse a partir de los textos y se compararán con otras variedades de la lengua.

\section{Metodología}

El corpus de referencia son los 31 cantos o collag que aparecen en "La poesía en los pueblos primitivos", de Alejandro Cañas Pinochet, publicado en 1908. Además, se tuvo a la vista "Estudios de la lengua veliche" de Cañas (1911), del cual procede un collag no publicado en el trabajo anterior. Finalmente, un verso perdido del mal llamado Collag del terremoto fue tomado de Lenz (1912).

El análisis de la fonética y fonología de la variedad de lengua registrada en los cantos se basa principalmente en los trabajos de Salas (1992), Smeets (2009), Sadowsky, Aninao, Cayunao y Heggary (2015) y en el Corpus Lexicográfico del Mapudungun (CORLEXIM) de Villena et al. (2016).

Para el análisis de la gramática y el léxico, se normalizó manualmente la escritura en una versión adaptada del Alfabeto Mapuche Unificado (SOCHIL, 1986). Los cambios con respecto a este alfabeto consisten en el uso de $<$ lh $>$ y $<$ nh $>$ en lugar de $<\underline{\text { l }}>$ y $<\underline{\text { n}}>$, por razones de legibilidad y tipografía; aparte de esto, se optó por usar $<\mathrm{v}>$ en vez $\mathrm{de}<\mathrm{f}>$, para alterar menos el texto y por motivos de representación fonética que se expon- 
drán en la discusión. En el caso de los variados grafemas empleados por Necul para representar consonantes laterales, se optó por simplificar la notación mediante el uso de $<$ ll $>$ para todo grafema que contuviera dos o más $<$ l $>$ y de $<$ l $>$ para todo grafema que tuviera al mismo tiempo $<$ l $>$ y $<$ r $>$ (incluso aquellos que tuvieran más de una $<\mathrm{l}>$ ), sin que esto signifique una presunción acerca de los fonemas que representaban estos símbolos.

La separación de palabras se hizo considerando las entradas del CORLEXIM y el Vocabulario de la lengua veliche (Cañas, 1911). Para segmentar sufijos, se siguió el análisis de Smeets (2009) y, secundariamente, el de Salas (1992).

\section{Resultados y discusión}

\subsection{Fonética y fonología}

Necul usó 56 grafemas para representar un idioma que, en otras variedades, posee entre 24 y 27 fonemas (Salas, 1992). Entre un collag y otro, Necul empleó diferentes escrituras para una misma palabra, en ocasiones incluso dentro de un mismo collag. En ciertos casos, estas diferencias son probablemente erratas de Necul o de la imprenta, como la alternancia entre quiñe y guiñe (kiñe, uno). Sin embargo, la inmensa mayoría de casos no pueden explicarse como erratas, sino que parecieran deberse a que durante su trabajo de recopilación Necul fue cambiando su ortografía o que intentó crear un protosistema de transcripción fonética del habla de sus informantes. En palabras de Lenz (1912, p. 759-760): "La escritura del indio muestra que é1 ha luchado heróicamente para vencer con su ortografia castellana las dificultades de la pronunciacion india, pero el resultado es poco satisfactorio para la lectura del que no sabe perfectamente la lengua".

De su escritura pueden deducirse algunos rasgos característicos de la pronunciación, mientras que en otros casos no es posible determinar la fonología subyacente. Se discutirá a continuación el inventario de vocales y la realización de las consonantes fricativas y laterales.

\subsubsection{Vocales}

El mapudungún posee seis vocales: /a, e, i, o, u, i/ (Salas, 1992), pero en la escritura de Necul tan solo aparecen <a, e, i, o, u>. En aquellos términos en que es esperable la sexta vocal $<\ddot{u}>$, aparecen en su lugar $<$ e $>$, como 
en gneman (=ngüman, llorar) ${ }^{3}$ o mel-llrreimí (=müleymi, estás); $<\mathrm{i}>$, como en aldi (=alhü, mucho) o anti (=anhtü, día), <u>, como en pudu (=püdu, pudú) o lllepun (=lepün, patio); o nada, como en ant (=anhtü, día) o tuguymn (=tuwimün, ustedes salen).

La ausencia de vocal al final de ant o entre m y n en tuguymn es relevante, pues la estructura silábica del mapudungún es (C)V(C) (Salas, 1992). Según Smeets (2009), si esta vocal está en una sílaba no acentuada se realiza como [ə], mientras que para Salas (1992), esta realización ocurre cuando la vocal está en el interior de la palabra o al final. De cualquier modo, ese alófono puede sonarle a un hispanohablante como ausencia de vocal (Salas, 1992) y ha sido frecuentemente omitido en la escritura en obras previas al siglo XX, como los diccionarios de Valdivia y Febrés (Villena et al., 2016).

Otro indicio de la distinción de seis vocales podría ser el uso de una consonante doble antes de $<$ e $>$, pues la combinación $<$ ppe $>$ aparece en tres palabras que en otras variedades poseen la sílaba /pì /: ppènhon (=pünon, pisada), ppel llomeñ (=püllomeñ, moscón azul) y ppeñeñ (=püñeñ, hijo de una mujer) (Villena et al., 2016).

En vista de lo anterior, es posible que la sexta vocal estuviera mucho más presente en el mapudungún de los collag de lo que da a entender a primera vista la escritura de Necul.

\subsubsection{Consonantes fricativas}

Las consonantes fricativas, representadas convencionalmente /f/ y / $\theta /$, tienden a realizarse como fricativas sonoras ([v], [ð]) en Arauco, Malleco, el Alto Biobío y Argentina, y como sordas ([f] y $[\theta]$ ) en otras zonas (Adelaar y Muysken, 2004). En el caso del chesüngun o huilliche de San Juan de la Costa y alrededores, Sadowsky et al. (2015) encontraron que los alófonos predominantes son $[\mathrm{f}]$ y $[\theta]$ respectivamente, pero que también aparecen $[\mathrm{h}],[\phi],[\mathrm{x}],[\mathrm{v}],[\mathrm{w}]$ y elisión para el primer caso, así como [ð], [f], [s], [ $]$ y elisión para el segundo. Este panorama tan heterogéneo del actual huilliche continental contrasta con los indicios que entrega la escritura de Necul para esas dos consonantes en Chiloé.

3 De aquí en adelante, los términos de Necul se indicarán en cursiva y entre paréntesis aparecerá su equivalente en mapudungún central según CORLEXIM y su glosa castellana. 
En el caso de /f/, Necul empleó en la mayor parte del texto la letra $<\mathrm{b}>$, por ejemplo en frases como "pibuimi pu cuñiball munchu", y algo menos $\langle\mathrm{v}\rangle$, usada sobre todo en el sufijo - vi, como en "peauvin tibu pel lle", mientras que $<\mathrm{f}>$ solamente aparece dos veces en el elemento querref (=kürüv, viento). Por lo tanto, es razonable pensar que se trataba de una consonante fricativa sonora, fuese $[v]$ o $[\beta]$, documentadas en variación libre en hablas como la de Tirúa (Salamanca y Quintrileo, 2009). Esto contradice hasta cierto punto la observación de Cavada (1914), quien afirmó que los indígenas alternaban los sonidos $\mathrm{de}<\mathrm{f}>\mathrm{y}<\mathrm{v}>$ al hablar castellano y decían, por ejemplo, "fotella" y "viesta”.

En cuanto a $/ \theta /$, el signo usado de forma consistente en todo el texto es $<\mathrm{d}>$, por ejemplo en domuche (=domuche, mujer), rraquiduam (=rakiduam, tristeza) o chinqued (=chüngküd, redondo). La combinación $<\mathrm{dd}>$ aparece una sola vez en el término quiddu (=kidu, solo). Para esta consonante, puede afirmarse con cierta seguridad que se habría tratado de una fricativa interdental sonora [ð], como la que existe en las variedades del norte de la actual área de distribución del mapudungún ya mencionadas.

Según Adelaar y Muysken (2004) y Zúñiga (2006), la consonante /s/ aparece en pocas raíces, en ningún morfema gramatical y normalmente procede de hispanismos. En este corpus en particular, aparece tan solo en hispanismos, en algunos casos integrados al sistema verbal mapuche. Su escritura es $<\mathrm{s}>\mathrm{o}<\mathrm{z}>$, según la ortografía castellana: "Dios", "camisa", “Cruz", "zarraza” (<“zaraza”), excepto en "rreboso” (< "rebozo").

\subsubsection{Consonantes laterales}

Según Adelaar y Muysken (2004), las consonantes laterales del mapudungún son la alveolar $/ 1 /$, la palatal $/ K /$ y la interdental $/ / /$. Según la literatura, la situación de estos fonemas en el huilliche continental sería inestable y se habrían producido innovaciones de diversa clase (Sadowsky et al., 2015).

La representación gráfica en los collag es notablemente heterogénea, pues Necul empleó 16 grafemas que contienen l: <l, l-1, l-1l, 1 ll, l-y, dl, ld, ll, ll-1, ll-1l, lll, 1-llr, 1-llrr, lrr, llrr, lllrr>. El uso de uno u otro signo no sigue una tendencia aparente, por ejemplo, la raíz müle-, "estar", aparece escrita melle, mel lle, mel-le y mel-llrre y la raíz lha-, "morir", aparece como dla o como la.

Es notable la baja frecuencia de $<\mathrm{l}>$ por sí sola, pues la mayoría de las palabras que en otras variedades poseen /l/ aquí aparecen representadas 
raramente con <l>: l-llamuen llrramuen (=lamngen, hermana), llepun l-llepun (=lepün, patio), melleldu (=mülelu, el que está), utrralepan (=wütralepan, estoy aquí de pie). Existe la posibilidad de que se hubiera producido un gran reajuste de las laterales, con tendencia hacia la palatalización, pero hay dos datos que hacen pensar que se trata de otra cosa. El primero es que los préstamos del mapudungún en el castellano chilote retienen la /l/, como puede verse en el Diccionario manual isleño de Cavada (1921), que contiene información sobre las etimologías. Esta objeción podría soslayarse si se postula que el cambio se produjo en el mapudungún de Chiloé después de que el término hubiera sido prestado. Sin embargo, hay un segundo dato que podría aclarar la notable escasez de $<\mathrm{l}>$ y consiste en que estos collag son el registro de cantos o recitaciones. En la lengua mapuche, se puede utilizar la palatalización como recurso para conferir un matiz afectuoso a lo que se dice (Salas, 1992) y este podría ser el caso, pues muchos de los cantos tratan de desdichas o de amistad. En la misma dirección apunta el uso de la partícula $\mathrm{em} \sim \mathrm{ema} \sim$ yem, que se utiliza para deplorar una situación pasada (Smeets, 2009).

El uso de $<\mathrm{dl}>$ y $<\mathrm{ld}>$ es consistente en la mayoría de los casos con la aparición de la consonante interdental / 1 / en otras variedades del idioma: dlahuen (=lhawenh, hierba medicinal), aldi (=alhü, mucho) y dla- (=lha-, morir). Pero también se utiliza en el sufijo verbal -ldu (=-lu) y en la raíz dladqui- (=lladkü, afligirse), lo cual puede ser un indicio de pérdida de contraste entre interdentales y alveolares. Sadowsky et al. (2015) postulan que uno de sus informantes posee un fonema bisilábico / $\underline{\mathrm{ld}} /$ como equivalente de esta consonante interdental. Sin más elementos no se puede proponer algo semejante para el mapudungún de Chiloé de fines del siglo XIX, pero es un dato a tener en cuenta. También, debe considerarse que Luis de Valdivia, en su diccionario de 1606, usaba en ocasiones la grafía $<\mathrm{ld}>$ para representar esta consonante, tradición y análisis que trascendió en el castellano en el préstamo "boldo" < volhu (Villena et al., 2016).

Los términos que tienen al mismo tiempo $<\mathrm{l}>\mathrm{y}<\mathrm{r}>$ son más difíciles de interpretar, pues sugieren la posibilidad de una lateral retrofleja [l] como la que planteó Salas para el huilliche en 1992, pero que Sadowsky et al. (2015) no atestiguaron en su reciente investigación. Estos signos aparecen de forma tan impredecible como la aparición de dobles o triples $<\mathrm{l}>$ antes descrita: llrramuen (=lamngen, hermana), mel-llrreimi (=müleymi, estás) y gnemalrrayan (=ngümalaan, no lloraré). Se puede 
postular lo mismo que en el caso anterior: una representación demasiado exhaustiva de la pronunciación de versos cantados. Sin embargo, también en el Vocabulario que contienen los Estudios de la lengua velice (Cañas, 1911) aparece varias veces esta combinación: quimellray (=kümelay, no es bueno) rlonco (=longko, cabeza), ul-lre (=wüle, mañana). Por ello, es plausible proponer que /1/ tenía un alófono con una realización retrofleja o incluso que se había creado una nueva oposición, aunque es virtualmente imposible determinar su naturaleza exacta.

\subsection{Gramática ${ }^{4}$}

$\mathrm{Al}$ segmentar morfemas, se hallaron los sufijos derivacionales (-chi, -we), el distributivo -ke, el pluralizador y locativo $p u$ y las posposiciones mo $\sim m u(=m e w)$ y pelle (=püle). En cuanto a los verbos, aparece una gran cantidad de sufijos verbales, característica bien conocida y comentada en diversos trabajos sobre el idioma (Salas, 1992; Adelaar y Muysken, 2004; Smeets, 2009). La lista de sufijos de Smeets (2009) supera los 100 elementos, mientras que en este corpus se encontraron 42, un número que dista de ser excepcional si se considera la baja frecuencia de algunos sufijos y la extensión del material analizado. A continuación, se expondrán dos características sobresalientes del uso de sufijos verbales.

\subsubsection{Interacciones entre primera y segunda persona}

Según Salas (1992) las interacciones entre primera y segunda persona se marcan de forma diferente en mapudungún central y en huilliche. En mapudungún central, se focalizaría siempre a la primera persona, sea agente o paciente: leli-e-n (me miraste), leli-e-yu (te miré), leli-mu-chi (mírenme), leli-w-iiñ (los miré/miramos a ustedes). En cambio, en huilliche se focalizaría siempre al paciente: leli-e-n, leli-e-ymi (te miré/miramos), leli-mu-chi, leli-e-ymün (los miré/miramos a ustedes). De este modo, los casos en que la segunda persona es agente y la primera paciente serían idénticos, pero al ser agente la primera persona y paciente la segunda, el sistema funcionaría al revés.

Las interacciones que aparecen en los collag son de los dos tipos, si se asume que hay un sufijo - $e$ oculto por procesos de fusión de vocales:

4 Abreviaturas: 1: primera persona, 2: segunda persona, 3: tercera persona, >: interacción agentepaciente, A: agente, BEN: benefactivo, CAUS: causativo, CVB: converbo, DUR: durativo, FUT: futuro, IMP: imperativo, IND: indicativo, ITJ: interjección, LOC: locativo, oвJ: objeto, PROX: proximidad, sG: singular, TR: transitivo. 
$\begin{array}{lllll}\text { (1) Quiñe } & \text { degnu } & \text { allquíll-lliayu } & \text { pínom } & \text { (Cañas, 1908, } p .156) \\ \text { Kiñe } & \text { dengu } & \text { allki-ll-ia-[e]-yu } & \text { piñom } & \\ \text { uno } & \text { asunto } & \text { oír-CAUS-FUT-[OBJ]-1SG>2SG.IND } & \text { querido } & \\ \text { Una } & \text { cosa } & \text { te haré oír } & \text { querido } & \end{array}$

(2) Huallgna acun molestapa peimi

ùlmen (Cañas, 1908, $p$.

$\begin{array}{llll}\text { Wall nga } & \text { aku-n } & \text { molesta-pa-pe-[e]-imi } & \text { ulmen } \\ \text { otra.vez ITJ } & \text { llegar.aquí-1SG.IND } & \text { molestar-PROX.LOC-PROX-[OBJ]-2SG.IND } & \text { señor } \\ \text { Otra vez } & \text { llegué aquí } & \text { a molestarte, } & \text { señor. }\end{array}$
141)

El sistema que documentó Luis de Valdivia en 1606 en Santiago y Félix de Augusta en 1903 entre los huilliches coincidía con (2), pues usaba - e y la terminación de la segunda persona paciente, sin marcar de ningún modo a la primera persona agente (Adelaar y Muysken, 2004). La coexistencia de estas dos formas en Chiloé podría indicar que la variedad insular se separó cuando era posible recurrir a los dos tipos de construcción para expresar estas interacciones.

También la interacción entre segunda persona agente y primera paciente aparece más de una posibilidad, como se verá a continuación:

(3) Quipal llelmochi

Kipa-ll-el-mo-chi

(Cañas, 1908, p. 146)

venir-CAUS-BEN-2A>1SG.IMP

Tráiganmelo

(4) Dihueñ-moechi

diweñ-mo-e-chi

(Cañas, 1908, p. 134)

acompañar-2A-OBJ-1SG.IMP

Acompáñenme

Desde la perspectiva del mapudungún central, lo esperable en (4) sería la terminación - $m u$-chi que aparece en (3) o -e-chi si el agente fuera la segunda persona singular, pero esta última forma en imperativo no se usa en el lenguaje hablado, sino la terminación en indicativo - $e-n$. (Salas, 1992)

Es posible que (4) fuera agramatical incluso para un hablante competente de Chiloé, pues los sufijos -mu y -e son mutuamente excluyentes. 


\subsubsection{Formas no finitas}

Es en el uso de formas no finitas donde los collag difieren más de otros textos en mapudungún pasados o actuales, como se verá a continuación:

Los sufijos - $n$, -lu, - $m$, - el, -fiel, -e-t-ew y -wma cumplen funciones equivalentes a las del modo infinitivo del castellano y aparecen en oraciones subordinadas y el predicado de frases nominales (Salas, 1992; Smeets, 2009).

En los collag no hay ningún caso de -e-t-ew ni de -wma, con excepción de la forma lexicalizada pe-wma (visión). Los demás aparecen con diferente frecuencia, siendo mayoritarios $-l u$ y $-m$.

Un uso anómalo de -lu es su aparición en formas conjugadas:

(5) Inaldu payeimi,

ina-lhu-pa-e-imi

seguir-CVB-PROX.LOC-OBJ-2SG.IND bus

Te seguí aquí, quintul-lupa yeymi

kintu-l-lu-pa-e-imi

buscar-TR-CVB-PROX.LOC-OBJ-2SG.IND te busqué aquí

$\begin{array}{lllll}\text { (6) De lig } & \text { cobque } & \text { niey queldu } & \text { (Cañas, 1908, p. I66) } \\ \text { De } & \text { lig } & \text { kovke } & \text { nie-i-ke-lhu } & \\ \text { ? } & \text { blanco } & \text { pan } & \text { tener-3SG.IND-HAB-CVB } & \\ \text { ? } & \text { pan } & \text { blanco } & \text { tiene } & \end{array}$

En (5) hay un paciente expreso y un agente implícito, mientras en (6) hay un verbo transitivo conjugado, pero además aparece -lu, en el primer caso incorporado y en el segundo como último elemento (-ke también está fuera de su posición esperable, pues todos los sufijos menos uno deben preceder al sujeto). Es posible que por cambio interno o por su función similar al gerundio castellano hubiera dado lugar a que se entendiera como un marcador de tiempo ( ${ }^{*}$ Estoy siguiéndote, estoy buscándote, *Tiene pan blanco ahora). Otra posibilidad es que los cantantes formaran palabras a su arbitrio, sin conocer del todo cómo operaban las frases subordinadas mapuches.

Las demás formas no finitas no aparecen en formas conjugadas, pero sí suele omitirse la expresión del posesivo o el pronombre independiente que requieren en las variedades vivas del idioma. Como antes, esto puede explicarse por el cambio lingüístico o por el desmantelamiento de la gramática de una lengua moribunda. 


\subsection{Léxico}

El texto publicado en 1908 es poco confiable en la separación de palabras, pues hay espacios y guiones entre morfemas o incluso dentro de morfemas. Sería necesario tener a la vista los originales para saber qué se debe a análisis de Necul, a decisión de Cañas o a errores de imprenta. La versión de 1911 es todavía menos confiable, pues le faltan versos y hay multitud de erratas en el texto. En este escenario, los resultados de la revisión del léxico no deben tomarse como definitivos.

El total de palabras del corpus es de 1232, de las cuales 504 son únicas (por ejemplo, utralepan y utrallepan se cuentan como dos). En este conjunto de palabras únicas, hay 245 formas verbales que proceden de 144 raíces. Salvando la distancia de la escritura y las posibles diferencias de fonología, se trata en su mayor parte de palabras reconocibles, si no todas de uso común, al menos la mayoría registradas en los diccionarios del CORLEXIM (Villena et al., 2016).

Algunas de estas palabras solo aparecen en los diccionarios más antiguos, pues han cambiado de forma o de significado. Por ejemplo, nawe, "hermana" según las traducciones de Cañas (1908), en el diccionario de Valdivia de 1606, se define como "muchacha de doce años abajo"; en el diccionario de Febrés de 1765, nawe ñawe es "hija del padre"; y en el de Augusta de 1916 es ñawe, "la hija (respecto al padre)" (Villena et al., 2016).

Unas pocas palabras no aparecen en estos diccionarios, pero sí en los Estudios de la lengua veliche (Cañas, 1911). Es el caso de diversos objetos del culto católico, al parecer sin equivalente en otras variedades de mapudungún: chinquedcoanti (=chingked-koanti, hostiario, lit.: redondo-?), huidimacuñ (=widi-makuñ, casulla, lit. olla-manta), ligthelque (=lig-trelke, papel, lit.: blanco-cuero) y malhuemilla (=malwe-milla, cáliz, lit.: vaso-oro).

Algunas pueden deducirse por el contexto, como lligpin, que parece equivaler a külleñü, lágrima. Finalmente, hay decenas de palabras que no pueden entenderse, como la raíz toro- o toropu-, que aparece cuatro veces en el corpus, pero en todos los casos la traducción que figura guarda escasa relación con el texto mapuche.

El léxico y también la gramática podrían comprenderse mejor si se trabajara con las traducciones originales de Necul, conservadas por Rodolfo Lenz en sus cuadernos (Labarías y Cárdenas, 1998). 


\section{Conclusiones}

El mapudungún que revelan los collag coincide en muchos puntos con otras variedades del idioma, tanto presentes como pasadas. Las diferencias fonéticas que sugiere la escritura pueden deberse en unos casos a la pronunciación del lenguaje cantado y en otros a procesos de cambio lingüístico. Las consonantes fricativas se pronunciaban como en variedades del norte y no como en el chesüngun o huilliche actual. En el caso de la gramática, se observa mayor distancia y pudiera pensarse que se debe al proceso de muerte del idioma, pues en la fecha de recolección del corpus ya no se trataba de una lengua de uso cotidiano en las islas del mar interior de Chiloé, sino que se restringía a la entonación de estos cantos y el recuerdo de algunos ancianos. El léxico, en cambio, es mayormente reconocible, a pesar de los siglos de separación.

La revisión del texto refrenda las críticas que hizo Rodolfo Lenz (1912) a las traducciones de Cañas y da cuenta de la importancia de revisar las traducciones originales de Necul que puedan haberse conservado en los papeles de Lenz.

\section{Bibliografía}

Adelaar, W. y Muysken, P. (2004). The Languages of the Andes. Cambridge: University Press.

Cañas, A. (1908). La poesía en los pueblos primitivos de América. Revista Chilena de Historia Natural, Año XII (1), 91-168.

(1911). Estudios de la lengua veliche. Santiago. En: C. Porter (ed.). Trabajos del Cuarto Congreso Científico ( $1^{\circ}$ Pan-Americano). Trabajos de la III Seción [sic] - Ciencias Naturales, Antropológicas y Etnológicas (pp. 143-330). Santiago de Chile: Imprenta Barcelona.

Cárdenas, R., Montiel, D., y Hall, C. (1991). Los chono y los veliche de Chiloé (pp. 203-205). Santiago de Chile: Olimpo.

Cárdenas, R. y Vásquez, J. (2008). Collags, poesía mapuche de Chiloé. Aproximaciones socioculturales a una compilación de poesía veliche hecha por Elias Necul de Caguach en 1887. Santiago de Chile: Ojoentinta.

Cavada, F. (1914). Chiloé y los chilotes. Santiago: Universitaria. 
. (1921). Diccionario manual isleño. Santiago de Chile: Yolanda.

Contreras, Constantino. (2009). Renato CÁRDENAS ÁLVAREZ y Jorge O. VÁSQUEZ V. Collags, poesía mapuche de Chiloé. Aproximaciones socioculturales a una compilación de poesía veliche hecha por Elías Necul de Caguach en 1887. Publicación de la Agrupación de Amigos Chachaén de la Biblioteca Pública de Achao: Imprenta Ojoentinta.

Labarías, M. y Cárdenas, J. (1998). Documentos auténticos de Rodolfo Lenz Católogo crítico. Cuadernos de la Facultad de Historia y Geografía. Santiago de Chile: Universidad Metropolitana de Ciencias de la Educación.

Lenz, R. (1912). Tradiciones e ideas de los araucanos acerca de los terremotos. Santiago de Chile: Cervantes.

Rondón, V. (2014). Havestadt v/s Febrés. A propósito de unas cartas y unas canciones. Revista de Historia Social y de las Mentalidades 18 (2), 79-103.

Sadowsky, S., Aninao, M., Cayunao, M. y Heggary, P. (2015). Huilliche: ¿Geolecto del mapudungun o lengua propia? Una mirada desde la fonética y la fonología de las consonantes. En A. Fernández y M. Regúnaga (eds.) Lingüistica indígena sudamericana: aspectos descriptivos, comparativos y areales (pp. 23-51). Buenos Aires: Editorial de la Facultad de Filosofía y Letras de la Universidad de Buenos Aires.

Salamanca, G. y Quintrileo, E. (2009). El mapuche hablado en Tirúa: Fonemas segmentales, fonotaxis y comparación con otras variedades. Revista de Lingüística Teórica y Aplicada 47 (1), 13-35.

Salas, A. (1992). El mapuche o araucano. Madrid: Mapfre.

Schuller, R. (1907). El vocabulario araucano de 1642-1643. Santiago: Cervantes.

SOCHIL (Sociedad Chilena de Lingüística). (1986) Encuentro para la unificación del alfabeto mapuche: Proposiciones y acuerdos. Temuco: Universidad Católica de Temuco.

Smeets, I. (2008). A grammar of Mapuche. Berlin - New York: Muyton de Gruyter.

Trivero, A. (1999). Inche ta Domitila Kuyul. Testimonio de la última Maestra de Paz de Chiloé. Ñuke Mapu. Recuperado de http:// www.mapuche.info/mapuint/Kuyul.html 
Villena, B., Chandía, A., Araya, I. y Flores, C. Corpus lexicográfico del mapudungún. <http://www.chandia.net/corlexim > o1 de julio de 2016.

Zúñiga, F. (2006). Mapudungun. El habla mapuche. Santiago de Chile: Centro de Estudios Públicos. 